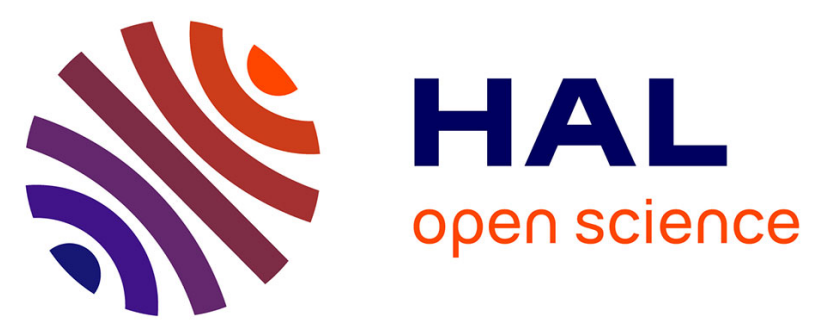

\title{
Fabrication of a Complex Two-Dimensional Adenine Perylene-3,4,9,10-tetracarboxylic Dianhydride Chiral Nanoarchitecture through Molecular Self-Assembly
} Xiaonan Sun, Manuela Mura, Harry T. Jonkman, Kantorovich N. Lev, Fabien Silly

\section{To cite this version:}

Xiaonan Sun, Manuela Mura, Harry T. Jonkman, Kantorovich N. Lev, Fabien Silly. Fabrication of a Complex Two-Dimensional Adenine Perylene-3,4,9,10-tetracarboxylic Dianhydride Chiral Nanoarchitecture through Molecular Self-Assembly. Journal of Physical Chemistry C, 2011, 116, pp.2493. 10.1021/jp2095054 . cea-00838027

HAL Id: cea-00838027 https://hal-cea.archives-ouvertes.fr/cea-00838027

Submitted on 24 Jun 2013

HAL is a multi-disciplinary open access archive for the deposit and dissemination of scientific research documents, whether they are published or not. The documents may come from teaching and research institutions in France or abroad, or from public or private research centers.
L'archive ouverte pluridisciplinaire HAL, est destinée au dépôt et à la diffusion de documents scientifiques de niveau recherche, publiés ou non, émanant des établissements d'enseignement et de recherche français ou étrangers, des laboratoires publics ou privés. 


\title{
Fabrication of Complex Two-Dimensional Adenine-PTCDA Chiral Nanoarchitecture through Molecular Self-Assembly
}

\author{
Xiaonan Sun, ${ }^{1}$ Manuela Mura, ${ }^{2}$ Harry T. Jonkman, ${ }^{1}$ Lev N. Kantorovich, ${ }^{2, *}$ and Fabien Silly ${ }^{3, \dagger}$ \\ ${ }^{1}$ Zernike Institute for Advanced Materials, \\ University of Groningen, Nijenborgh 4, \\ NL-9747 AG Groningen, The Netherlands. \\ ${ }^{2}$ Physics, King's College London, The Strand, \\ London, WC2R 2LS, United Kingdom \\ ${ }^{3}$ CEA, IRAMIS, SPCSI, Hybrid Magnetic Nanoarchitectures, F-91191 Gif-sur-Yvette, France
}

(Dated: June 24, 2013)

\begin{abstract}
This document is the unedited author's version of a Submitted Work that was subsequently accepted for publication in Journal of Physical Chemistry C, copyright American Chemical Society after peer review. To access the final edited and published work see http://pubs.rsc.org/en/journals/journalissues/tc.
\end{abstract}

The two-dimensional self-assembly of nonsymmetric adenine DNA base mixed with symmetric PTCDA molecules is investigated using scanning tunneling microscopy (STM). We experimentally observe that these two building blocks form a complex close-packed chiral supramolecular network on $\mathrm{Au}(111)$. The unit cell of the adenine-PTCDA nanoarchitecture is composed of 14 molecules. The high stability of this structure relies on PTCDA-PTCDA and PTCDA-adenine hydrogen bonding. Detailed theoretical analysis based on the Density functional theory (DFT) calculations, reveals that adenine molecules work as a "glue" providing additional strengthening to the PTCDA-based skeleton of this sophisticated multicomponent nanoarchitecture. At the same time, we find that orientation and chirality of Adenine molecules across the monolayer is likely to vary leading to a disorder in the atomistic structure of the entire assembly. 


\section{INTRODUCTION}

Tailoring complex organic nanoarchitectures is the focus of recent research interest for developing novel nanostructured materials ${ }^{1}$. Two-dimensional (2D) molecular nanoarchitectures can be engineered taking advantage of molecular self-assembly ${ }^{2}$. These structures can be tailored at the nanometer scale by exploiting intermolecular interactions. Molecules forming hydrogen bonds $(\mathrm{H}-$ bonds) are particularly interesting building blocks for creating sophisticated organic architectures ${ }^{3}$ due to high selectivity and directionality of these bindings. Various single ${ }^{4}$ and multicomponent ${ }^{5}$ H-bonded structures have been created using semiconducting as well as biomolecules ${ }^{6}$.

Engineering new molecular bioarchitectures is especially appealing for developing new advanced drug delivery devices and sensors. Engineering multicomponent biomaterials via attaching one molecule to another requires deep understanding of molecular interactions. Molecular building blocks have to be chemically and structurally compatible to form specific structures. Over the last decade a remarkable progress has been achieved in building on crystal surfaces molecular assemblies. This has been largely based on a 'drop and see' approach rather on the understanding of the underlying processes and interactions. It is widely believed that a more intelligent approach is now required which would allow "design" or "dial" a structure with specific properties. Molecular size and geometry, as well as interaction between molecules are some of the key parameters determining whether or not molecular self-assembly is possible, and what the final outcome will be. For instance, it has been shown that the mixture of adenine and cytosine, both DNA bases, does not lead to the formation of ordered multicomponent structures ${ }^{7}$ whereas several multicomponent structures have been obtained using semiconducting perylene-3,4,9,10tetracarboxylic-3,4,9,10-dianhydride (PTCDA) molecules mixed pentacene ${ }^{8}$ and 2,4,6-Triamino1,3,5-triazine, sym-Triaminotriazine ${ }^{9}$.

In this paper we investigate the self-assembly of nonsymmetric adenine DNA base (Fig. 1(a)) mixed with symmetric PTCDA molecules (Fig. 1(b)) on $\mathrm{Au}(111)-(22 \times \sqrt{3})$ surface at room temperature in ultra high vacuum. We observed using scanning tunneling microscopy (STM) that the molecules form a complex bicomponent chiral supramolecular network. The unit cell of the 2D PTCDA-adenine architecture is composed of 14 molecules. The high stability of this architecture relies on PTCDA-PTCDA and PTCDA-adenine hydrogen bonding. Density functional theory (DFT) modeling reveals that PTCDA based skeleton of this complex nanoarchitecture is stabilized further by the adenine molecules which may take several orientations within the unit cell rendering 
the whole monolayer to be adenine-disordered.

\section{EXPERIMENTAL SECTION}

The substrates were $\mathrm{Au}(111)$ films grown on mica. The samples were introduced into the ultrahigh vacuum (UHV) chamber of an STM (Omicron STM equipped with Nanonis controller) operating at a pressure of $10^{-7} \mathrm{~Pa}$, and were then sputtered with argon ions and annealed at $500^{\circ} \mathrm{C}$. Molecules were sequentially deposited onto a room temperature gold surface. PTCDA molecules (Fig.1(b)) were sublimated at $260^{\circ} \mathrm{C}$ followed by adenine (Fig.1(a)) sublimated at $150{ }^{\circ} \mathrm{C}$. Cut PtIr wire was used as STM tip to obtain constant current $\left(\mathrm{I}_{t}\right)$ images at room temperature with a bias voltage $\left(\mathrm{V}_{s}\right)$ applied to the sample. STM images have been processed and analyzed using FabViewer ${ }^{10}$.

\section{RESULTS AND DISCUSSION}

\section{Experimental observation of Adenine-PTCDA Self-Assembly on Au(111) Surfaces}

Figure 1(c) shows an STM image of the $\mathrm{Au}(111)-(22 \times \sqrt{3})$ surface after sequential deposition of PTCDA and adenine molecules onto the surface at room temperature. The semiconducting PTCDA molecules and adenine DNA bases self-assemble into a complex 2D chiral architecture (Fig. 1(c,d)) which drastically differs from pure herringbone or brick-wall PTCDA structure ${ }^{11}$ and pure adenine networks ${ }^{7}$. The STM image in Fig. 1(c) reveals the reconstructed Au(111)- $(22 \times \sqrt{3})$ surface next to the adenine-PTCDA supramolecular network. The gold cristaline directions are indicated by black arrows in Fig. 1(c).

PTCDA molecules appear brighter than adenine molecules in the STM images. The unit cell of the PTCDA-adenine network is represented in Fig. 1(e,f) by a yellow dashed lines; it has a shape of a parallelogram with $4.1 \mathrm{~nm}$ and $3.4 \mathrm{~nm}$ sides and the acute angle of $\sim 75^{\circ}$ between them. This organic network is chiral, with two enantiomeric domains presented in the STM images of Fig. 1(e) and (f). The unit cell of this multicomponent two-dimensional nanoarchitecture consists of 14 molecules, namely of 10 PTCDA and 4 adenine molecules.

\section{Modeling of Adenine-PTCDA two-dimensional architecture}

The atomistic model of the supramolecular self-assembly is presented in Fig. 1(g). The net- 
work is composed of rows of PTCDA molecules connected with each other through two O $\cdots \mathrm{H}-\mathrm{C}$ hydrogen bonds (along the red dashed arrows in Fig. 1(g). These molecules form a template or external skeleton (called the Ske structure in the following) shown separately in Fig. 2(a); the skeleton has rather large pores. A square composed of four PTCDA molecules connected with each other in a domino-like manner is located in the full structure of Fig. 1(g) in the center of each pore of the Ske structure. The four PTCDA molecules in the square are connected to each other

a

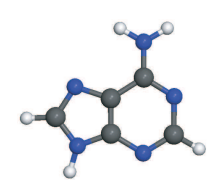

C

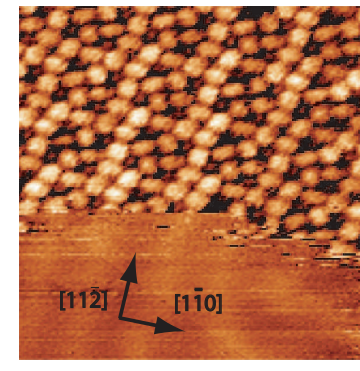

e

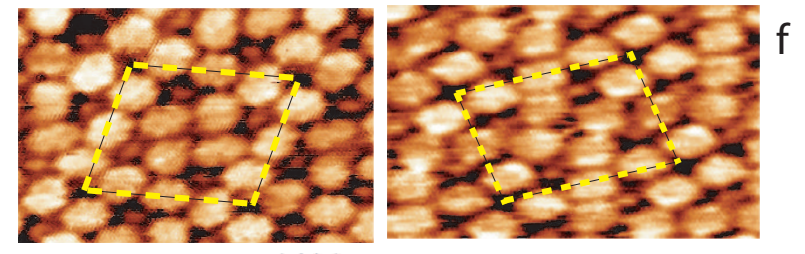

B.

9

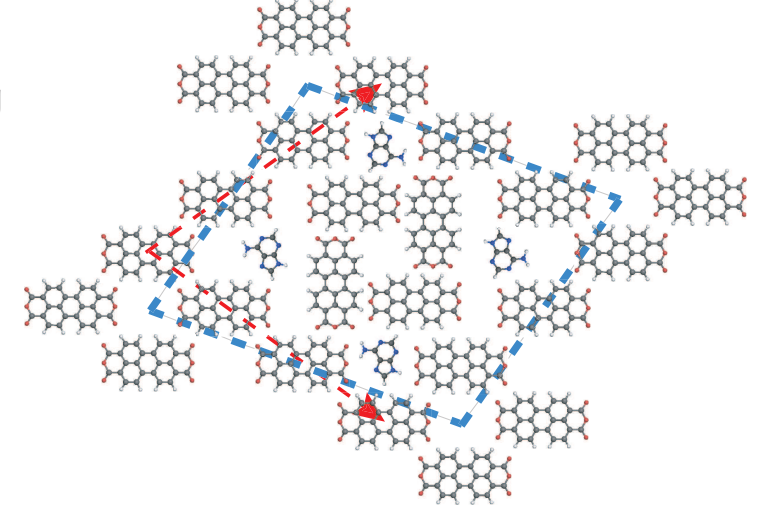

FIG. 1: (a) Adenine (9H-purin-6-amine) and (b) PTCDA (3,4,9,10-perylenetetracarboxylic-dianhydride) molecules, where carbon, oxygen, hydrogen and nitrogen atoms are gray, red, white, and blue, respectively. The PTCDA-adenine self-assembled chiral network on the $\mathrm{Au}(111)-(22 \times \sqrt{3})$ surface: (c) $18 \times 18 \mathrm{~nm}^{2}$; $\mathrm{V}_{s}=-0.5 \mathrm{~V}, \mathrm{I}_{t}=0.4 \mathrm{nA}$, (d) $11 \times 11 \mathrm{~nm}^{2} ; \mathrm{V}_{s}=-0.8 \mathrm{~V}, \mathrm{I}_{t}=0.4 \mathrm{nA}$. The two enantiomeric domains are presented in the high-resolution STM images: (e) $5 \times 3 \mathrm{~nm}^{2} ; \mathrm{V}_{s}=-0.5 \mathrm{~V}, \mathrm{I}_{t}=0.4 \mathrm{nA}$ and (f) $5 \times 3 \mathrm{~nm}^{2} ; \mathrm{V}_{s}$ $=-0.8 \mathrm{~V}, \mathrm{I}_{t}=0.4 \mathrm{nA}$. The unit cell is indicated by a dashed yellow lines. $(\mathrm{g})$ Model of the PTCDA-adenine nanoarchitecture. 
through three $\mathrm{O} \cdots \mathrm{H}-\mathrm{C}$ hydrogen bonds. The external skeleton with squares of domino-attached four PTCDA molecules is shown in Fig. 2(b). Finally, four adenine molecules contribute to the supramolecular network unit cell. They are connected to PTCDA molecules through O $\cdots \mathrm{H}-\mathrm{N}$, $\mathrm{O} \cdots \mathrm{H}-\mathrm{C}$ and $\mathrm{N} \cdots \mathrm{H}-\mathrm{C}$ hydrogen bonds. In total, the multicomponent unit cell consists of 14 molecules: 10 PTCDA and 4 adenine molecules. In comparison, the unit cell of pure PTCDA network is composed of 2 molecules $^{11}$ and the one assembled by pure adenine is composed of 2 or 4 molecules ${ }^{12}$. Our results show that mixing symmetrical and nonsymmetrical molecules could be a method to increase the sophistication of hydrogen-bonded supramolecular architectures.

In the case of single-component architectures, hydrogen-bonds can lead to the formation of a large number of adenine ${ }^{12}$ and many hydrogen-bonded PTCDA networks ${ }^{11}$. In all these structures homodimers are the basic building blocks that can lead to numerous single-component networks with various numbers of molecules in the unit cell. In comparison, the adenine-PTCDA network shown in Fig. 1(d) is far more complex: the unit cell is composed of a large number of molecules which are connected to each other in a sophisticated manner. Note that only a single structure has been observed (also see below).

To understand the stability of this network, density functional calculations (DFT) have been performed in the gas-phase approximation (without presence of the surface) which was demonstrated to be appropriate for these molecules and the gold surface ${ }^{13}$. Four configurations have been considered. The first configuration is composed of six PTCDA molecules in the unit cell, see Fig. 2(a), and serves to reproduce the periodicity of the external skeleton. The second and the third structures are composed of ten molecules in the unit cell: in one case we added to the skeleton structure four PTCDA molecules in the centre of the skeleton in the domino-like geometry, Fig. 2(b), and in the other case four adenine molecules at the corners of the skeleton were added instead, Fig. 3(a). Finally, the full configuration composed of all fourteen molecules in the unit cell that can reproduce the whole network observed in the STM images has been considered, and the DFT relaxed structure is shown in Fig. 3(b).

The first structure that we analyze is the skeleton (Ske) structure composed of solely six PTCDA molecules, see Fig. 2(a). This structure serves as a template for all structures to be discussed below and eventually for the final assembly. The stabilization energy of the relaxed structure is $-1.34 \mathrm{eV}$ as shown in Table I. This corresponds to $-0.22 \mathrm{eV}$ per PTCDA molecule and is of the same order of magnitude as the values found in Ref. ${ }^{11}$ for some other PTCDA structures. The lattice vectors and the angle between the lattice vectors in this relaxed structure are somewhat larger that those 

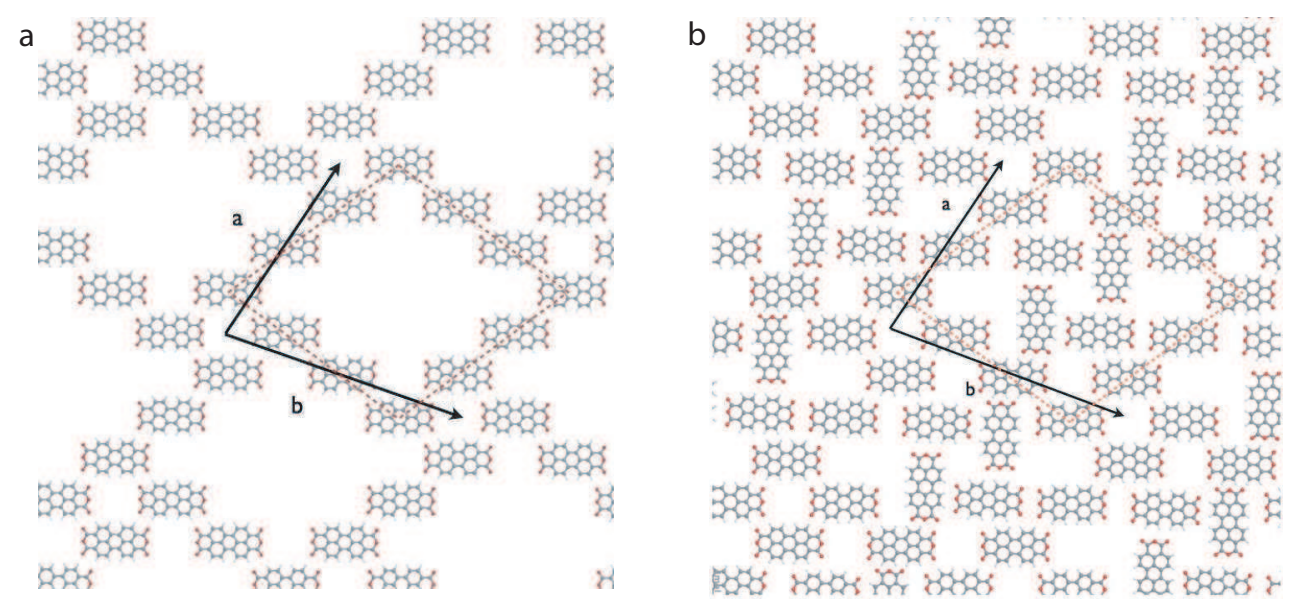

FIG. 2: (a) The DFT relaxed structure of the external skeleton, referred to as Ske in the text. It contains 6 molecules in the unit cell. (b) The DFT relaxed structure (referred to as Ske+PTCDA) of the external skeleton with added internal domino-like squares, containing 10 molecules in the unit cell.

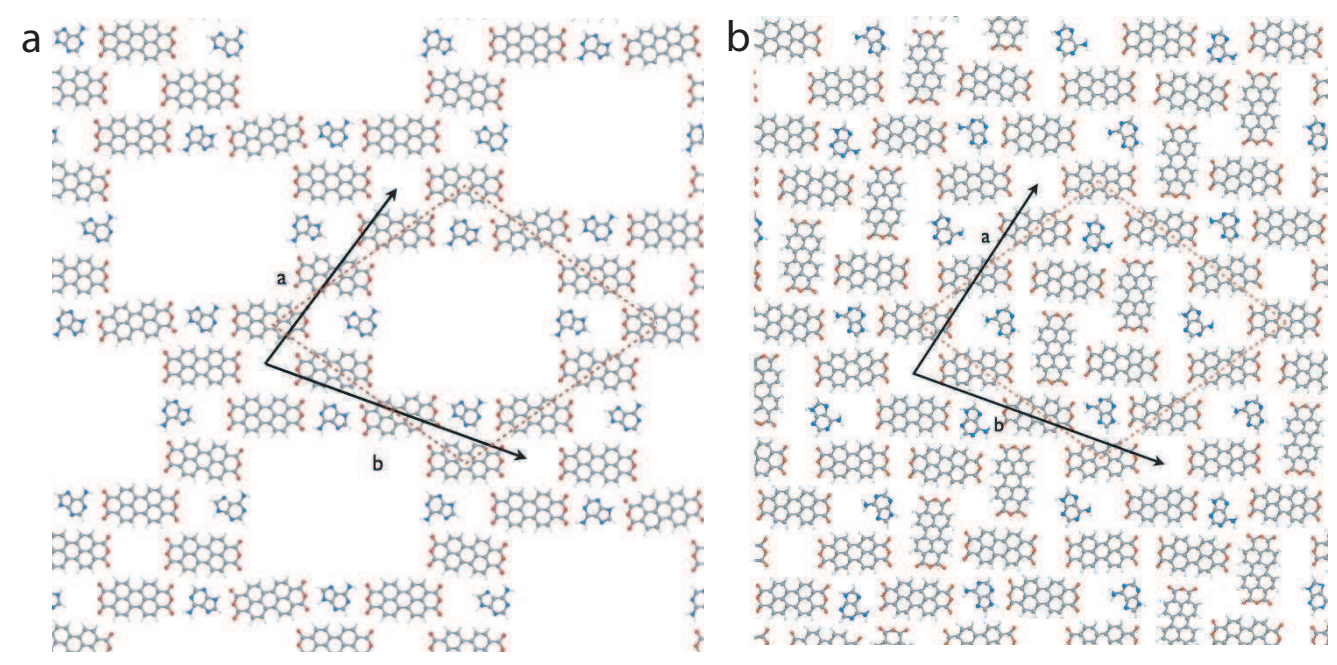

FIG. 3: (a) The DFT relaxed structure of the external skeleton and added adenine molecules with 10 molecules in the unit cell, referred to as Ske+Adenine. (b) The complete structure: the external skeleton with internal squares of PTCDA molecules and adenine molecules added in pores of it. Altogether, there are 14 molecules in the unit cell. The structure shown has been obtained using geometry relaxation with our DFT method; the unit cell and the lattice vectors are also indicated.

experimentally observed for the whole assembly, see Table II. If four PTCDA molecules are added to the skeleton structure in a domino-like manner in the same way as in the final structure, see the structure Ske+PTCDA in Fig. 2(b), the stability per molecule is increased by almost $0.1 \mathrm{eV}$, see Table I. However, if instead of the square composed of four PTCDA molecules one adds to the skeleton structure four adenine molecules as shown in Fig. 3(a), this structure (to be refereed to as Ske+Adenine) on the whole becomes slightly more energetically favorable as the binding energy 
per molecule increases by additional $0.03 \mathrm{eV}$ (both structures Ske+PTCDA and Ske+Adenine have 10 molecules in the cell). One can see that adding four PTCDA molecules to the skeleton brings in 8 additional H-bonded "contacts" to the structure; the same number of additional contacts are created if four adenine molecules are added instead; however, in this latter case the H-bonds are relatively stronger due to presence of $\mathrm{N}-\mathrm{H}$ groups in the adenine which form stronger $\mathrm{H}$-bonds with oxygen atoms of the PTCDA molecules. Interestingly, in all these cases the deformation energies are found to be negligible (see Table I). Adding both PTCDA square and adenine molecules to the skeleton structure, Fig. 3(b), stabilizes the final structure even more although the energy gain per molecule is rather small.

If the squares of PTCDA molecules serve mainly to fill in the empty space in the large pores of the skeleton structure, adenine molecules serve to stabilize the whole construction. This can be seen from the density difference plot of the final structure shown in Fig. 4. Alternating regions of depletion and excess of the density along the $\mathrm{H}$ bonds, the so-called "kebab" structures (see computational details), characterize the strength of the $\mathrm{H}$ bonding. If the kebab structures in contacts between PTCDA molecules are underdeveloped and hence correspond to weak H-bonds, one can clearly see well developed kebab structures formed between adenine and PTCDA molecules which correspond to much stronger H-bonds ${ }^{15}$.

The lattice vectors of every structure we considered are compared with those obtained in the experiment for the whole assembly in Table II. The calculated lattice vectors and the angle between them for the skeleton structure are somewhat larger than those observed in our STM experiments for the whole structure. Additional PTCDA and adenine molecules added in the pores of the template expand the lattice vectors even more, although adenine molecules seem to bring some reduction in the angle. On the whole, the relaxed final geometry of the entire network is in a reasonable agreement with the STM observations. The obtained discrepancy may be explained by the neglect of the non-local correlation effects or van der Waals (vdW) interaction between molecules in our calculations; this extra attraction which is especially important to consider between weakly bounded PTCDA molecules (see discussion in Ref. ${ }^{13}$ ), is expected to result in some contraction of the lattice. One cannot exclude here also the effect of the surface which has not been accounted for in our simulations.

Note that in the STM image of this system, Fig. 1(d), the exact orientations of the adenine molecules and their chirality (adenine is prochiral as opposite to PTCDA) can not be unambiguously determined, since the molecules appear as much smaller dim features compared to the well 


\begin{tabular}{|c|c||c|c|c|}
\hline \multicolumn{2}{|c||}{ Ske } & Ske+PTCDA & Ske+Adenine & whole \\
\hline$E_{\text {stab }}$ & -1.34 & -3.03 & -3.33 & -4.37 \\
$E_{B S S E}$ & 1.43 & 2.20 & 1.79 & 2.48 \\
$E_{\text {def }}$ & 0.06 & 0.04 & 0.08 & 0.05 \\
$E_{\text {int }}$ & -1.41 & -3.07 & -3.41 & -4.41 \\
\hline
\end{tabular}

TABLE I: Calculated stabilization, $E_{\text {stab }}$, deformation, $E_{\text {def }}$, and interaction, $E_{\text {int }}$, energies for the four considered structures. The BSSE correction is significant in each case and is also given.

\begin{tabular}{|c|c||c|c|c|c|}
\hline \multicolumn{2}{|c|}{ Ske } & Ske+PTCDA & Ske+Adenine & whole & experiments \\
\hline $\mathrm{a}$ & 3.7 & 3.7 & 3.6 & 3.7 & 3.4 \\
$\mathrm{~b}$ & 4.2 & 4.4 & 4.5 & 4.5 & 4.1 \\
$\beta$ & 76.2 & 77.1 & 74.4 & 75.9 & 75 \\
\hline
\end{tabular}

TABLE II: Computed lengths $a$ and $b$ of the lattice vectors (in $\mathrm{nm}$ ) and the acute angle $\beta$ between them (in degrees) for the four structures considered.

resolved rectangular PTCDA molecules. As usual, theoretical calculations should have come to rescue as they indicate the most favorable orientations of the four adenine molecules within the pores created by surrounding PTCDA molecules. Although the geometry shown in Figs. 3(b) and 4 corresponds to the total energy minimum and hence may indicate the expected geometry of adenines, a closer examination shows that in fact a number of possibilities exist here for adenine orientations. We notice that the four adenine molecules may be evenly split into two

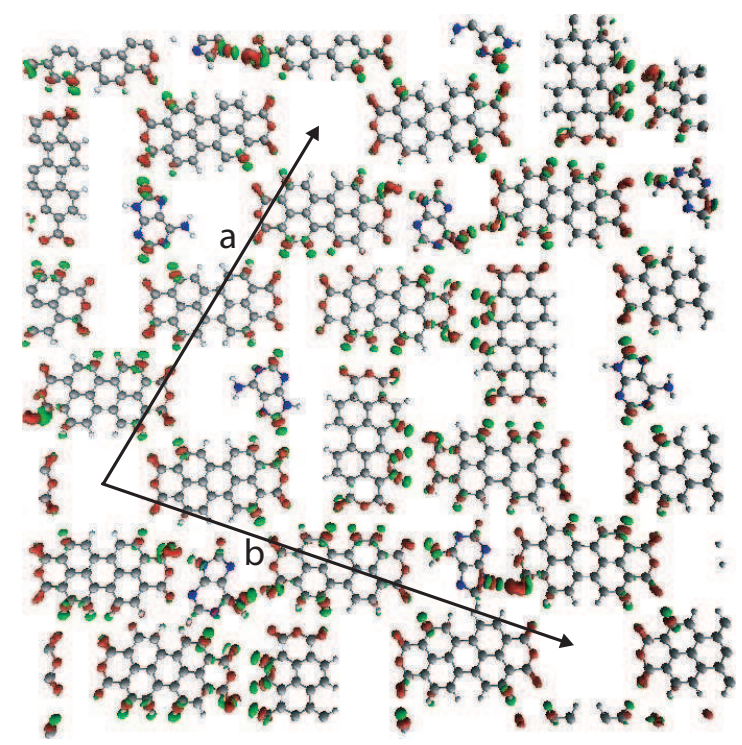

FIG. 4: The electron density difference plot (corresponding to \pm 0.01 electrons / $\AA^{3}$ ) of the complete PTCDA and adenine monolayer. Green colour corresponds to excess, while red colour to depletion of the electron density. 
groups corresponding to two distinct types of surroundings created by PTCDA molecules around them, see Fig. 3(b). In order to understand the orientation of adenine molecules we performed some additional calculations on characteristic finite clusters composed of six PTCDA and one adenine molecule. One such surrounding (out of the two mentioned above) formed by six PTCDA molecules and shown in Fig. 5 is considered as an example here. In the configuration considered PTCDA molecules are placed as in the original structure and a single adenine molecule is added in the pore as shown; in total there are seven molecules in the unit cell. Two chiralities of adenine are possible and both were investigated. Moreover, different orientations of the adenine inside the pore for both chiralities were investigated by rotating the adenine molecule with respect to the surrounding PTCDA molecules and performing the corresponding constrained geometry relaxation, and the most favorable geometries found are shown in Fig. 5(a) and (b). In all these calculations selected carbon atoms of the PTCDA molecules were fixed in the geometry of the skeleton structure; also the carbon atoms of the adenine were fixed to keep the chosen rotation of the molecule; all other degrees of freedom were allowed to relax. We find that for the chirality of the adenine shown in Fig. 5(a) there are two distinct minima: one, shown in the Figure is by about $0.2 \mathrm{eV}$ lower in energy than the other one which corresponds to an additional $60^{\circ}$ clockwise rotation of the adenine molecule. In both cases the adenine makes a single $\mathrm{H}$ bond to either of the two PTCDA molecules. In the case of the opposite chirality, shown in Fig. 5(b), there is only one distinct energy minimum corresponding to the geometry shown; it is by nearly $0.4 \mathrm{eV}$ more favorable than several others which are quite close in energy between them. In all cases the barriers separating different minima are quite large - around $0.7 \mathrm{eV}$. The most favorable geometries shown in Fig. 5 involve hydrogen bonds between the N-H groups of the adenine and the oxygen atoms of the PTCDA which are known to be quite strong ${ }^{14-18}$. The high stability of these configurations is illustrated in the electron density difference plots in Fig. 5(a,b), where the well defined kebab structure of the adenine-PTCDA bonds is clearly seen compared to the weak PTCDA-PTCDA bonds. The structures shown in Figs. 3(a) and 3(b) correspond to a particular orientation of the adenine molecules (which is in fact not the most energetically favorable).

These results indicate that there is a certain degree of randomness or disorder in the orientation and chirality of the four adenine molecules within each unit cell as well as in different cells; because of that, strictly speaking, the whole structure is not periodic, only the substructure formed by the PTCDA molecules can be approximately considered periodic. This disorder however was not possible to identify in our STM images in which adenine molecules are seen as dim protrusions. 

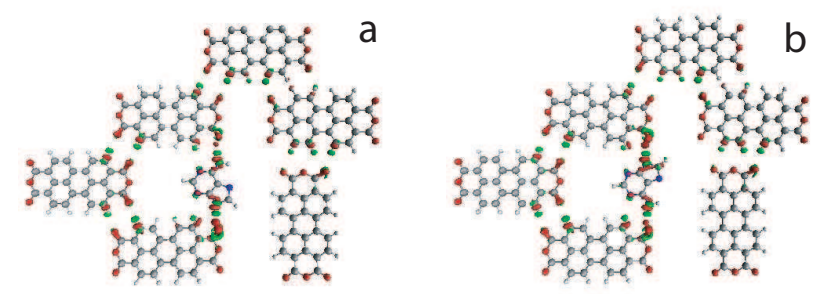

FIG. 5: $(a, b)$ Two geometries of the six PTCDA molecules and adenine relaxed with our DFT method. The two structures correspond to two possible chiralities of the adenine. The electron density difference plot of adenine and five PTCDA molecules corresponds to \pm 0.01 electrons / $\AA^{3}$ shows regions of access (green) and depletion (red) of the electron density.

We experimentally explored the stability of this $2 \mathrm{D}$ nanoarchitecture to validate our calculations. We observed that surface post annealing does not lead to the formation of other AdeninePTCDA structures. We also did not observe that variation of the adenine-PTCDA ratio deposited on the surface affects the adenine-PTCDA self-assembly. We indeed observed that high concentration of adenine (as compared to PTCDA) leads to appearance of local defects induced by adenine molecules which completely segregate from the same adenine-PTCDA structure as discussed above (Fig. 6). These observations also support our calculations in that, i.e. the observed architecture appears to be formed preferentially and that adenine possible orientations weakly affects the stability of the structure.

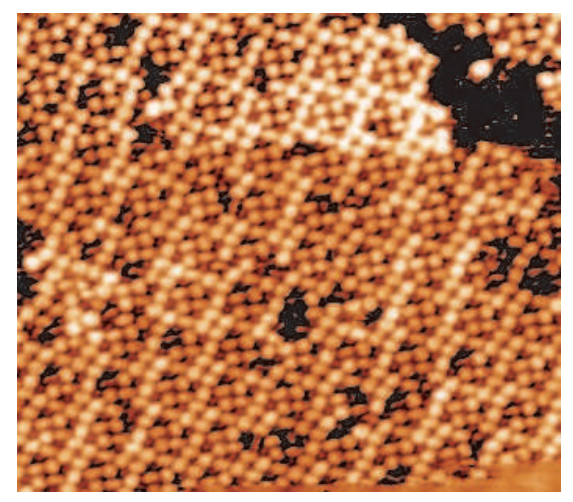

FIG. 6: STM image of the PTCDA-adenine self-assembly on $\mathrm{Au}(111)-(22 \times \sqrt{3})$ surface (area $35 \times 30 \mathrm{~nm}^{2}$; $\mathrm{V}_{s}=-0.4 \mathrm{~V}, \mathrm{I}_{t}=0.4 \mathrm{nA}$ ) observed after deposition of higher adenine to PTCDA ratio.

\section{CONCLUSION}

In this paper we have investigated the self-assembly of PTCDA and adenine on the $\mathrm{Au}(111)$ surface at room temperature. We show using scanning tunneling microscopy that PTCDA and 
adenine self-assemble into a sophisticated supramolecular architecture, which unit cell is composed of 14 molecules. A detailed theoretical analysis based on the ab initio DFT calculations, revealed that adenine molecules work as a "glue" providing additional strengthening to the PTCDA network. At the same time, there is a certain freedom with which adenine molecule may fill in the pores between the PTCDA molecules leading to the partially disordered network. These results illustrate that complex multi-component structures can be engineered by mixing symmetric and nonsymmetric organic molecules on crystal surfaces. We believe that this route in design of complex structures may result in new exciting assemblies with interesting properties.

\section{Computational Details}

The calculations were performed using the ab-initio SIESTA method ${ }^{19}$, which is based on localized numerical orbital basis set, periodic boundary conditions, and the first principles scalarrelativistic norm-conserving Troullier-Martins ${ }^{20}$ pseudo-potential factorized in the KleinmanBylander $^{21}$ form. We used Perdew, Becke and Ernzerhof $(\mathrm{PBE})^{22}$ generalized gradient approximation for the exchange and correlation which was found previously to be adequate in representing hydrogen bonding between DNA base molecules ${ }^{14}$. In each calculation, atomic relaxation was performed until forces on atoms were less than $0.01 \mathrm{eV} / \AA$ in the cases of dimers and $0.03 \mathrm{eV} / \AA$ in the cases of monolayers.

A number of energies are worked out and used in the analysis. Firstly, the stabilization energy, $E_{\text {stab }}$, is defined as the total energy of the relaxed combined system (e.g. the PTCDA network) minus the total energies of all its individual components (PTCDA molecules and adenine) relaxed separately. The BSSE correction must be applied to this energy to account for the fact that the localized basis set is used; the counterpoise method has been used here to correct for this ${ }^{23}$. The system is considered stable if $E_{\mathrm{stab}}<0$. To characterize the interaction between the parts of a composite system (e.g. a pair), the interaction energy, $E_{\text {int }}$, is used, which is defined as the energy of the pair minus the energy of each individual molecule calculated in the geometry of the pair (i.e. without relaxing them individually); this energy is always negative for a stable system. Finally, the deformation energy, $E_{\text {def }}$, characterizes the total energy lost by all parts of the combined system (e.g. by the two molecules of the pair) due to their subsequent relaxation in the composite system (e.g. the pair). It is calculated as a sum of differences between the energies of individual molecules in the combined system and when they are completely separated (at infinity), $E_{i}^{0}$ : 


$$
E_{\mathrm{def}}=\sum_{i}\left(E_{i}-E_{i}^{0}\right)
$$

The interaction and deformation energies, as defined above, must sum up exactly to the stabilization energy, i.e. $E_{\text {stab }}=E_{\text {int }}+E_{\text {def }}$. In this way, one can see how the deformation energy is compensated by the interaction energy if the structure is indeed stable. Note, however, that this relationship would only be exact if plane waves were used in our DFT calculations. Since, we use SIESTA which employs a localized basis set, this relationship would only be exact prior to the application of the BSSE correction. Therefore, to simplify the analysis, the same BSSE correction was applied to the interaction and stabilization energies ensuring that this exact relationship remains.

The stabilization energy gives an indication of the strength of the hydrogen bonds in the system. The relaxed geometry also provides an indirect indication about the stability of the hydrogen bonds enabled in the structure: the hydrogen bonds often prefer planar configurations and there are preferential values in the hydrogen bonds of the donor-H-acceptor distances and of the angle associated with them ${ }^{14-18}$; a general rule is that hydrogen bonds try to become as linear as possible with the acceptor-H-donor distance, depending on the actual acceptors involved, being within the range of 2.6-3.0 $\AA$.

Another way of characterizing the strength of the hydrogen bonding between e.g. two molecules is by analyzing the electron density difference plots ${ }^{11,14-18,24,25}$. Hydrogen bonds were found to display the so-called "kebab" structure of alternating regions of charge excess and depletion, that represent the redistribution of the charge density due to the hydrogen bond formation ${ }^{14}$. The stronger the bond, the more "regular" the "kebab" structure is. This concept can easily be generalized for complexes containing more than two molecules.

\section{ACKNOWLEDGMENT}

The research leading to these results has received funding from the European Research Council under the European Union's Seventh Framework Programme (FP7/2007-2013) / ERC grant agreement $\mathrm{n}^{\circ}$ 259297. XS acknowledges a Bernoulli Fellowship from the University of Groningen. M.M. would like to thank for the computer time allocation on the HPCx and HECToR U.K. 
National facilities via the Material Chemistry consortium.

* Electronic address: lev.kantorovitch@kcl.ac.uk

$\dagger$ Electronic address: fabien.sillyecea.fr

1 (a) Yang, Y.; Wang, C. Chem. Soc. Rev. 2009, 38, 2576-2589.

(b) Yu,M.; Kalashnyk, N.; Xu, W.; Barattin, R. Benjalal, Y.; Laegsgaard, E.; Stensgaard, I.; Hliwa, M.; Bouju, X.; Gourdon, A.; Joachim, C.; Besenbacher, F.; Linderoth, T. R. ACS Nano 2010, 4, 4097-4109. (c) Whittell, G. R.; Hager, M. D; Schubert, U. S.; Manners, I. Nat. Mater. 2011, 10, 176-188.

(d) Klyatskaya, S.; Klappenberger, F.; Schlickum, U.; Kühne, D.; Marschall, M.; Reichert, J.; Decker, R.; Krenner, W.; Zoppellaro, G.; Brune, H.; Barth, J. V.; Ruben, M. Adv. Funct. Mater. 2011, 21, 12301240.

(e) Lu, C.; Zhu, E.; Liu, Y.; Liu, Z.; Lu, Y.; He, J.; Yu, D.; Tian, Y.; Xu, B. J. Phys. Chem. C 2010, 114, 3416-3421.

2 (a) Liang, H.; Sun, W.; Jin, X.; Li, H.; Li, J.; Hu, X.; Teo, B. K.; Wu, K. Angew. Chem. Int. Ed. 2011, 50, 7562-7566. (b) Uemura, S.; Aono, M.; Komatsu, T.; Kunitake, M. Langmuir 2011, 27, 1336-1340. (c) Jensen, S.; Greenwood, J.; Früchtl, H. A.; Baddeley, C. J. J. Phys. Chem. C 2011, 115, 8630-8636. (d) Palma, C.-A.; Bjork, J.; Bonini, M.; Dyer, M. S.; Llanes-Pallas, A.; Bonifazi, D.; Persson, M.; Samori, P. J. Am. Chem. Soc. 2009, 131, 13062-13071. (e) Deak, D. S.; Silly, F.; Porfyrakis, K.; Castell, M. R. Nanotechnology 2007, 18, 075301. (f) Baris, B.; Luzet, V.; Duverger, E.; Sonnet, P.; Palmino, F.; Cherioux, F. Angew. Chem. Int. Ed. 2011, 50, 4094-4098.

3 (a) Gardener, J. A.; Shvarova, O. Y.; Briggs, G. A. D.; Castell, M. R. J. Phys. Chem. C 2010, 114, 58595866. (b) Jensen, S.; Greenwood, J.; Früchtl, H. A.; Baddeley, C. J. Phys. Chem. C 2011, 115, 86308636. (c) Silly, F.; Shaw, A. Q.; Briggs, G. A. D.; Castell, M. R. Appl. Phys. Lett. 2008, 92, 023102. (d) Walch, H.; Maier, A.-K.; Heckl, W. M.; Lackinger, M. J. Phys. Chem. C 2009, 113, 1014-1019.

4 (a) Yu, M.; Kalashnyk, N.; Barattin, R.; Benjalal, Y.; Hliwa, M.; Bouju, X.; Gourdon, A.; Joachim, C.; Laegsgaard, E.; Besenbacher, F.; Linderoth, T. R. Chem. Commun. 2010, 46, 5545. (b) Mura, M.; Silly, F.; Briggs, G. A. D.; Castell, M. R.; Kantorovich, L. N. J. Phys. Chem. C 2009, 113, 21840-21848.

5 (a) de Oteyza, D. G.; Barrena, E.; Dosch, H.; Ortega, J. E.; Wakayama, Y. Phys. Chem. Chem. Phys. 2011, 13, 4220. (b) Liang, H.; Sun, W.; Jin, X.; Li, H.; Li, J.; Hu, X.; Teo, B. K.; Wu, K. Angew. Chem. Int. Ed. 2011, 50, 7562-7566. (c) Walch, H.; Maier, A.-K.; Heckl, W. M.; Lackinger, M. J. Phys. Chem. 
C 2009, 113, 1014-1019. (d) Nath, K. G.; Ivasenko, O.; MacLeod, J. M.; Miwa, J. A.; Wuest, J. D.; Nanci, A.; Perepichka, D. F.; Rosei, F. J. Phys. Chem. C 2007, 111, 16996-17007. (e) Silly, F.; Weber, U. K.; Shaw, A. Q.; Burlakov, V. M.; Castell, M. R.; Briggs, G. A. D.; Pettifor, D. G. Phys. Rev. B 2008, 77, 201408.

6 Shen, H.-Y.; Liu, Y.-Q.; Gao, J.; Zhen, H.-M.; Zhu, N.; Li, J. DNA and Cell Biology 2011, 30, 255-264.

7 Otero, R.; Xu, W.; Lukas, M.; Kelly, R. E. A.; Laegsgaard, E.; Stensgaard, I.; Kjems, J.; Kantorovich, L. N.; Besenbacher, F. Angew. Chem. Int. Ed. 2008, 47, 9673-9676.

8 Chen, W.; Li, H.; Huang, H.; Fu, Y.; Zhang, H. L.; Ma, J.; Wee, A. T. S. J. Am. Chem. Soc. 2008, 130, 12285-12289.

9 Sun, X.; Jonkman, H. T.; Silly, F. Nanotechnology 2010, 21, 165602.

10 Silly, F. J Microsc-Oxford 2009, 236, 211-218.

11 Mura, M.; Sun, X.; Silly, F.; Jonkman, H. T.; Briggs, G. A. D.; Castell, M. R.; Kantorovich, L. N. Phys. Rev. B 2010, 81, 195412

12 Lukas, M.; Kelly, R. E. A.; Kantorovich, L. N.; Otero, R.; Xu, W.; Laegsgaard, E.; Stensgaard, I.; Besenbacher, F. J. Chem. Phys. 2009, 130, 024705.

13 Mura, M.; Gulans, A.; Thonhauser, T.; Kantorovich, L. Phys. Chem. Chem. Phys. 2010, 12, 4759.

14 Kelly, R. E. A.; Lee,Y. J.; Kantorovich, L. N. J. Phys. Chem. B 2005, 109, 22045.

15 Kelly, R. E. A.; Kantorovich, L. N. J. Mater. Chem. 2006, 16, 1894.

16 Kelly, R. E. A.; Lee,Y. J.; Kantorovich, L. N. J. Phys. Chem. B 2005, 109, 11933.

17 Kelly, R. E. A.; Lee,Y. J.; Kantorovich, L. N. J. Phys. Chem. B 2006, 110, 2249.

18 Kelly, R. E. A.; Kantorovich, L. N. J.Phys. Chem. C 2007, 111, 3883.

19 Sanchez-Portal, D. ; Ordejon,P. ; Artacho,E. ; Soler, J. M. Int. J. Quant. Chem. 1997, 65, 453-461.

20 Troullier,N. ; Martins,J. L. Phys. Rev. B 1991, 43, 1993-2006.

21 Kleinman, L. Phys. Rev. B 1980, 21, 2630.

22 Perdew, J. P.; Burke,K.; Ernzerhof, M. Phys. Rev. Lett. 1996, 77, 3865.

23 Boys, F.; Bernardi, F. Mol. Phys. 1970, 19, 553-566.

24 Silly, F.; Shaw, A. Q.; Castell, M. R.; Briggs, G. A. D.; Mura, M.; Martsinovich, N.; Kantorovich, L. J. Phys. Chem. C 2008, 112, 11476-11480.

25 Mura, M.; Martsinovich, N.; Kantorovich, L. Nanotechnology 2008, 19, 465704. 